\title{
On Dirichlet series and Hecke triangle groups of infinite volume
}

\author{
by \\ Marvin I. Knopp (Philadelphia, Penn.) and \\ MARK Sheingorn (New York, N.Y.)
}

We present a construction of infinitely many linearly independent solutions, in Dirichlet series $\varphi(s)$, of Riemann's functional equation,

$$
R(k-s)=R(s), \quad R(s)=(2 \pi / \lambda)^{-s} \Gamma(s) \varphi(s) .
$$

Here $k$ is an arbitrary real number (the weight) and $\lambda>2$, but otherwise unrestricted. The proof entails the use of the Riemann-Hecke-Bochner correspondence between modular integrals on the one side, and Dirichlet series with functional equations, on the other. It requires, as well, an application of Eichler's generalized Poincaré series. Since $\lambda>2$ here, the underlying group of invariance, the Hecke triangle group

$$
G_{\lambda}:=\left\langle\left(\begin{array}{cc}
1 & \lambda \\
0 & 1
\end{array}\right),\left(\begin{array}{cc}
0 & -1 \\
1 & 0
\end{array}\right)\right\rangle
$$

is of the second kind; that is, $G_{\lambda}$ has a fundamental region of infinite volume. This necessitates a new approach to the proof of convergence of the generalized Poincaré series. In particular, it requires estimates for certain word lengths in the Hecke group of the second kind $(\lambda>2)$, in terms of the trace. In fact, by generalizing ideas of Eichler, we present such estimates for arbitrary finitely generated Fuchsian groups of the second kind.

1. Introduction. The first author's previous article [8] presents a construction of infinitely many linearly independent solutions of Riemann's functional equation

$$
R\left(\frac{1}{2}-s\right)=R(s), \quad R(s)=\pi^{-s} \Gamma(s) \varphi(s),
$$

1991 Mathematics Subject Classification: Primary 11F66, 11F67.

The second-named author thanks the NSA, NSF, and PSC-CUNY for supporting this research. 
with $\varphi(s)$ a Dirichlet series convergent in some right half-plane. While at first glance this appears to violate Hamburger's well-known theorem on the uniqueness of Riemann's $\zeta(s)[5,6,12]$, the results of [8] obtain under weaker auxiliary conditions than those assumed in Hamburger's theorem(s). (For a detailed explanation see the introductions to [6] and [8].)

The final section of [8] describes two natural extensions, the first resulting from replacement of the functional equation (1) by

$$
R(k-s)=R(s), \quad R(s)=\pi^{-s} \Gamma(s) \varphi(s),
$$

where $k$ is an arbitrary real number. This generalization can be derived with no essential alteration in the methods of [8]. (However, there is a distinction between the cases $k<2$ and $k \geq 2$.) The second extension involves generalizing the factor $\pi^{-s}$ to $(2 \pi / \lambda)^{-s}, \lambda>0$, in the definition of $R(s)$. Thus, instead of (2), we treat

$$
R_{\lambda}(k-s)=R_{\lambda}(s), \quad R_{\lambda}(s)=(2 \pi / \lambda)^{-s} \Gamma(s) \varphi_{\lambda}(s),
$$

with $\varphi_{\lambda}(s)$ a Dirichlet series convergent in some right half-plane.

In (3) the relevant values of $\lambda$ are of two kinds:

$$
\begin{aligned}
& \lambda=2 \cos \pi / p, \quad p \in \mathbb{Z}, p \geq 3 ; \\
& \lambda \geq 2 .
\end{aligned}
$$

In [8] we have dealt exclusively with the case $\lambda=2$, i.e. $(2 \pi / \lambda)^{-s}=\pi^{-s}$; the subject of the present article is the case $\lambda>2$, with $\lambda$ otherwise arbitrary. This case presents substantial new difficulties which do not occur in [8]. These arise because, in contrast to the situation in [8], the Hecke group $G_{\lambda}$ (defined in (6), below) has "free sides" - and thus infinite volume - when $\lambda>2$. The new problems are of two kinds:

- The construction of [8] involves division by Eisenstein series attached to $G_{\lambda}$. That these may well have an infinite number of zeroes in the upper half plane $\mathcal{H}$, accumulating at the free sides, invalidates the approach of [8].

- The modified approach applied here, like that of [8], rests upon the use of Eichler's "generalized Poincaré series" $[4,10,11]$ to obtain "many" linearly independent automorphic integrals for $G_{\lambda}$. (See the definitions given in (27) of Section 4 and in Section 3, following the statement of Theorem 2.) However, all previous discussions of Eichler's generalized Poincaré series have been restricted to groups of finite volume. In particular, all proofs of convergence of these series have required Eichler's estimates of word length in the group, and his proof relies strongly on the assumption of finite volume $[4, \S 3]$.

To overcome the first difficulty we apply results of $[7, \S 6]$ : when $\lambda>2$ there exist Poincaré series bounded away from zero in a fundamental region of $G_{\lambda}$. (This is impossible when $\lambda \leq 2$.) To deal with the second problem, 
we obtain (in Section 6) analogues of Eichler's estimates for the groups $G_{\lambda}, \lambda>2$. (Section 7 treats arbitrary infinite-volume finitely generated Fuchsian groups.)

The method of [8] requires, in addition to the generalized Poincaré series, Bochner's generalization to automorphic integrals of the familiar RiemannHecke correspondence between modular forms and Dirichlet series with functional equations [2]. Application of the correspondence here entails no new problems in the change from $\lambda=2$ to $\lambda>2$, since in [2] Bochner worked with arbitrary $\lambda$ (Section 2, below).

The invariance group of the automorphic integrals appearing in the correspondence is

$$
G_{\lambda}=\left\langle S_{\lambda}, T\right\rangle, \quad S_{\lambda}=\left(\begin{array}{cc}
1 & \lambda \\
0 & 1
\end{array}\right), \quad T=\left(\begin{array}{cc}
0 & -1 \\
1 & 0
\end{array}\right) .
$$

$M=\left(\begin{array}{ll}a & b \\ c & d\end{array}\right)$ acts on $\mathcal{H}$ as a Möbius transformation: $M z=(a z+b) /(c z+d)$. With $\lambda$ of the types given in (4) and (5) the $G_{\lambda}$ are called the Hecke (triangle) groups; these are precisely the values of $\lambda$ for which $G_{\lambda}$ is discrete. If $\lambda \geq 2$, $G_{\lambda}$ has the single relation

$$
T^{2}=I \text {. }
$$

For $\lambda$ of the type given in (4), on the other hand, there is the second relation

$$
\left(S_{\lambda} T\right)^{p}=I \text {. }
$$

When $G_{\lambda}$ is discrete, the set

$$
\mathcal{R}_{\lambda}=\{z \in \mathcal{H}|| z|>1,| \Re z \mid<\lambda / 2\}
$$

is a fundamental region for $G_{\lambda}$. When $\lambda>2, \mathcal{R}_{\lambda}$ has "free sides", and thus infinite volume.

As already indicated, the free sides of $\mathcal{R}_{\lambda}$ force us to turn to the results of [7] for the construction of modular integrals. However, [7] deals with "absolute" Poincaré series, rather than the "relative" parabolic Poincaré series of Petersson. This entails the application here of the absolute Eichler generalized Poincaré series in place of the relative generalized Poincaré series discussed in [10] and utilized in [8]. The series we require is a modified and generalized version of that developed by Lehner in $[11, \S 3]$.

As it turns out, once the problem of word length is resolved, the fact that $\mathcal{R}_{\lambda}$ has free sides makes it possible to strengthen the results of [8] in carrying them over to the case $\lambda>2$. Specifically, beyond constructing infinitely many linearly independent solutions of (3), we in fact prove a "Mittag-Leffler" theorem for Dirichlet series with functional equation:

Theorem 1. Let $k$ be any real number, $\lambda>2$ and $A(s)$ any rational function such that $A(k-s)=A(s)$. Then there exists a Dirichlet series $\varphi_{k, \lambda}(s)=\varphi(s)$, convergent in some right half-plane such that 
$P(s)$;

(i) $P(s) \varphi(s)$ is an entire function of finite order for some polynomial

(ii) the function $R_{k, \lambda}(s)=(2 \pi / \lambda)^{-s} \Gamma(s) \varphi(s)$ satisfies $R_{k, \lambda}(k-s)=$ $R_{k, \lambda}(s)$;

(iii) $R_{k, \lambda}(s)-A(s)$ is entire.

Remark. Except for the assertion that $P(s) \varphi(s)$ has finite order, (iii) implies (i).

2. The Riemann-Hecke-Bochner correspondence. Bochner [2] formulated the general correspondence; Chandrasekharan and Narasimhan have applied it extensively in [3]. It appears as well in $\S 2$ of [8] and we include a statement here once again in Lemmas 1 and 2 below. The correspondence is essential to our proof, reducing the question of existence of the Dirichlet series $\varphi_{k, \lambda}(s)$ of Theorem 1 to a question of existence of a function $F_{k, \lambda}(z)$ holomorphic in $\mathcal{H}$, and with transformation properties resembling those of an automorphic form of weight $k$ on $G_{\lambda}$. (We have called such a function $F_{k, \lambda}$ an "automorphic integral" on $G_{\lambda}$; see $\S 3$ of [8].) As in [8] we complete the proof of Theorem 1 by constructing such $F_{k, \lambda}$ through the use of generalized Poincaré series.

Lemma 1. Let $k$ be real, $C$ complex and $\lambda_{1}, \lambda_{2}>0$. Suppose $F(z)$ and $G(z)$ are functions holomorphic in the upper half-plane $\mathcal{H}$, defined there by the exponential series

$$
F(z)=\sum_{n=0}^{\infty} a_{n} e^{2 \pi n z / \lambda_{1}}, \quad G(z)=\sum_{n=0}^{\infty} b_{n} e^{2 \pi n z / \lambda_{2}},
$$

with $a_{n}$ and $b_{n}$ satisfying

$$
a_{n}, b_{n}=O\left(n^{\gamma}\right), \quad \gamma>0, n \rightarrow \infty .
$$

Let $\Phi(s)$ and $\Psi(s)$ be the Mellin transforms of $F(i y)-a_{0}$ and $G(i y)-b_{0}$, respectively:

$$
\begin{aligned}
& \Phi(s)=\int_{0}^{\infty}\left\{F(i y)-a_{0}\right\} y^{s} \frac{d y}{y}=\left(\frac{2 \pi}{\lambda_{1}}\right)^{-s} \Gamma(s) \sum_{n=1}^{\infty} a_{n} n^{-s}, \\
& \Psi(s)=\int_{0}^{\infty}\left\{G(i y)-b_{0}\right\} y^{s} \frac{d y}{y}=\left(\frac{2 \pi}{\lambda_{2}}\right)^{-s} \Gamma(s) \sum_{n=1}^{\infty} b_{n} n^{-s} .
\end{aligned}
$$

Then the assertions (A) and (B) are equivalent:

(A) For $z \in \mathcal{H}$,

$$
z^{-k} G(-1 / z)=C F(z)+q(z),
$$


where $q(z)$ is a "log-polynomial sum",

$$
q(z)=\sum_{1 \leq j \leq J} z^{\alpha_{j}} \sum_{0 \leq t \leq M(j)} \beta(j, t)(\log z)^{t} .
$$

(In (14), $\alpha_{1}, \ldots, \alpha_{j}$ are complex numbers, as are the coefficients $\beta(j, t)$. The $t$ are nonnegative integers.)

(B) $\Phi$ and $\Psi$ can be continued analytically to the entire s-plane, except for a finite number of poles. Furthermore, $\Phi$ and $\Psi$ are bounded in each "lacunary" vertical strip:

$$
\sigma_{1} \leq \Re s \leq \sigma_{2}, \quad|\Im s| \geq t_{0}>0,
$$

and they satisfy the functional equation

$$
\Psi(k-s)=e^{\pi i k / 2} C \Phi(s) .
$$

Remarks. (i) The lemma differs somewhat from Bochner's original formulation in [2], which deals with generalized Dirichlet series, rather than the ordinary Dirichlet series we have here. Furthermore, Bochner's period functions $q(z)$ in the modular relation (13) are "residual functions" - in his terminology - rather than the sums (14). (See also [3].) However the sums (14) are residual in Bochner's sense; moreover, a residual function occurring as a period function in a modular relation (13) necessarily has the form (14).

(ii) The discrete group relevant to Theorem 1 is $G_{\lambda}$. In the proof of the Theorem we apply Lemma 1 with $\lambda_{1}=\lambda_{2}=\lambda, k$ an arbitrary real number and $F=G$ (thus $\Phi=\Psi$, as well). With $F=G$, the modular relation (13) states that $F$ is a "modular integral" of weight $k$ on $G_{\lambda}$, with "period function" $q(z)$. (See Section 3 for the definitions.)

Lemma 1 omits any mention of the relationship between the period function $q(z)$ and the Dirichlet series in statement (B). In fact, $q(z)$ determines the exact locations and orders of the poles of $\Phi$ and $\Psi$, and vice versa. We make this precise for the special case $F=G, \Phi=\Psi$, in

Lemma 2. Suppose $F=G, \Phi=\Psi$, in Lemma 1. Then the term $\beta z^{\alpha}(\log z)^{t}, \beta \neq 0, t \in \mathbb{Z}, t \geq 0$, occurring in (14) corresponds to poles of $\Phi(s)$ of order $t+1$ at the points $s=\alpha+k$ and $s=-\alpha$. The only possible further singularities of $\Phi$ are simple poles at $s=0$ and $s=k$.

3. Multiplier systems and cocycles for $G_{\lambda}$. The application of Lemma 1-with $\lambda_{1}=\lambda_{2}=\lambda, F=G, \Phi=\Psi$-shows that Theorem 1, our main result, is equivalent to

Theorem 2. Suppose $k$ is real and $C=e^{-\pi i k}$. Let $r(z)$ be any logpolynomial sum (14), and form the new log-polynomial sum

$$
q(z)=e^{\pi i k / 2} z^{-k} r(-1 / z)-r(z) .
$$


Then there exists a function

$$
F(z)=\sum_{n=0}^{\infty} a_{n} e^{2 \pi i n z / \lambda}, \quad z \in \mathcal{H},
$$

with

$$
a_{n}=O\left(n^{\gamma}\right), \quad \gamma>0, n \rightarrow \infty,
$$

such that for $z \in \mathcal{H}$,

$$
e^{\pi i k / 2} z^{-k} F(-1 / z)=F(z)+q(z) .
$$

Remarks. (i) By the growth restriction, (18), $F(z)$ is holomorphic in $\mathcal{H}$. A function $F$ satisfying (17) and (19) is called an automorphic integral on $G_{\lambda}$, of weight $k$ with period function $q(z)$. Of course, if $q(z) \equiv 0$, then $F$ is an automorphic form of weight $k$ on $G_{\lambda}$.

(ii) A function $q(z)$ occurring in (19) necessarily satisfies

$$
e^{\pi i k / 2} z^{-k} q(-1 / z)+q(z) \equiv 0,
$$

since $T z=-1 / z$ has order 2 . The condition (20) is equivalent, in turn, to the expression (16) for $q(z)$. This underlies the restriction of the $q(z)$ in Theorem 2 to those log-polynomial sums defined by an expression of the form (16).

(iii) The condition (20) is the basis for the assertion in Lemma 2 that a single term in $q(z)$ gives rise to two poles in $\Phi(s)$. Note that the poles of $\Phi(s)$ must occur in pairs if

$$
\Phi(k-s)=\Phi(s), \quad s \in \mathbb{C} .
$$

(The condition (21) is simply (15) for the case $\Psi=\Phi, C=e^{-\pi i k / 2}$. It differs from condition (ii) of Theorem 1 only in notation.)

In order to describe the version of the generalized Poincare series applicable to Theorem 2, we begin with a fixed log-polynomial sum $q(z)$ satisfying (20). We shall use $q(z)$ to generate a "cocycle" $\left\{q_{M} \mid M \in G_{\lambda}\right\}$, and the cocycle to construct the appropriate generalized Poincaré series.

First, we define a suitable multiplier system in weight $k$ for $G_{\lambda}$, that is, a set of complex numbers $\left\{v(M) \mid M \in G_{\lambda}\right\}$, with $|v(M)|=1$ for all $M \in G_{\lambda}$ and satisfying the weight $k$ "consistency condition":

$$
v\left(M_{3}\right)\left(c_{3} z+d_{3}\right)^{k}=v\left(M_{1}\right) v\left(M_{2}\right)\left(c_{1} M_{2} z+d_{1}\right)^{k}\left(c_{2} z+d_{2}\right)^{k} .
$$

The identity (22) is required to hold for all $z \in \mathcal{H}, M_{1}, M_{2} \in G_{\lambda}$, with

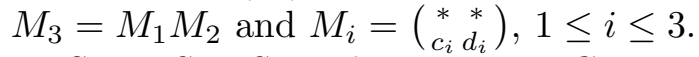

Since $S=S_{\lambda}$ and $T$ generate $G_{\lambda}$, it is clear that (22) provides a means of defining a multiplier system $v(M)$ on $G_{\lambda}$ once we specify $v(S)$ and $v(T)$. This is subject only to the proviso that these numbers be consistent with the relations on $G_{\lambda}$. But for $\lambda>2$ the only relation in $G_{\lambda}$ is (7). Consequently, 
this leaves $v(S)$ unrestricted and, by a simple calculation with (22), forces $v(T)$ to be one of the four complex numbers $\pm e^{-\pi i k / 2}, \pm i e^{-\pi i k / 2}$. As it turns out, the choices $\pm i e^{-\pi i k / 2}$ both lead to trivial spaces of automorphic forms on $G_{\lambda}$, of weight $k$. For our application we choose $v(S)=1$ and $v(T)=e^{-\pi i k / 2}(=C$, the number of Theorem 2$)$.

Given the real number $k$ and the multiplier system $v$ in weight $k$, we define the slash operator

$$
\left(\left.f\right|_{k} ^{v} M\right)(z)=\bar{v}(M)(c z+d)^{-k} f(M z)
$$

for $M=\left(\begin{array}{cc}* & * \\ c & d\end{array}\right) \in G_{\lambda}$ and any function $f$ defined in $\mathcal{H}$. A cocycle on $G_{\lambda}$, for weight $k$ and multiplier system $v$, is any collection of $\left\{q_{M} \mid M \in G_{\lambda}\right\}$, defined on $\mathcal{H}$ and satisfying the "cocycle condition"

$$
q_{M_{1} M_{2}}=\left.q_{M_{1}}\right|_{k} ^{v} M_{2}+q_{M_{2}}, \quad M_{1}, M_{2} \in G_{\lambda} .
$$

As with the consistency condition (22) for multiplier systems, the condition (24) will yield a cocycle for $G_{\lambda}$ once $q_{S}$ and $q_{T}$ are specified, provided only that $q_{S}$ and $q_{T}$ are consistent with relation (7) in $G_{\lambda}$.

It is easy to check that (7) leaves $q_{S}$ unrestricted and requires of $q_{T}$ only that it satisfy

$$
\left.q_{T}\right|_{k} ^{v} T+q_{T}=0
$$

However, by our definition of $v,(25)$ is precisely the same as (20), hence equivalent to the condition (16). Thus we may generate a cocycle $\left\{q_{M} \mid M\right.$ $\left.\in G_{\lambda}\right\}$ with the choice

$$
q_{S}=0, \quad q_{T}=q,
$$

where $q$ is given by (16).

4. Eichler's generalized Poincaré series. The series we require in the proof of Theorem 2 is

$$
\Psi(z)=\Psi\left(\left\{q_{M}\right\}, f, m ; z\right)=\sum_{V} \frac{q_{V}(z) f(V z)}{[(\alpha z+\beta)+i(\gamma z+\delta)]^{2 m}}
$$

where the summation is over all $V=\left(\begin{array}{ll}\alpha & \beta \\ \gamma & \delta\end{array}\right) \in G_{\lambda}$. We assume $f$ is bounded in $\mathcal{H}$ and $m \in \mathbb{Z}^{+}$is a "large" integer. Here $\left\{q_{M}\right\}$ is the cocycle generated by (24) and (26) from the log-polynomial sum $q(z)$ given by (16) of Theorem 2 , as described in Section 3. (Note that, although the notation does not reflect it directly, $\Psi$ depends upon the weight $k$ and the corresponding multiplier system $v$, since $\left\{q_{M}\right\}$ does.)

Let $\mathcal{P}$ denote the collection of all functions $\varphi$ holomorphic in $\mathcal{H}$, satisfying the growth condition

$$
|\varphi(z)| \leq K\left(|z|^{\alpha}+y^{-\beta}\right), \quad y=\Im z>0,
$$


for some constants $K, \alpha, \beta>0$. This space is relevant here since the coefficients $\left\{a_{n}\right\}$ of an exponential series $F(z)$ defined by (17) satisfy the restriction (18) if and only if $F \in \mathcal{P}$. This observation will enter into the proof of Theorem 2 at the conclusion of the argument (Section 5).

For H-groups (finitely generated Fuchsian groups of the first kind having at least one cusp - which we normalize as $i \infty)$, it is shown in [10, pp. 615-619], following Eichler [4], that for $m$ sufficiently large the relative generalized Poincaré series

$$
\sum_{V} \varrho_{V}(z)(\gamma z+\delta)^{-2 m}, \quad V=\left(\begin{array}{cc}
* & * \\
\gamma & \delta
\end{array}\right),
$$

converges uniformly on compact subsets of $\mathcal{H}$. In $(29),\left\{\varrho_{V}\right\}$ is a cocycle for the group in question, with $\varrho_{V} \in \mathcal{P}$ for all $V$, and the summation is over all $V$ in the group with distinct lower rows $\gamma, \delta$.

Since $q_{V} \in \mathcal{P}$ for the cocycle generated by (24), (26) it is clear that the series (27) and (29) are closely related. Thus, the method employed in [10] (and [11], where a special case of (27) — not (29) — is treated) to deal with the series (29) may be adapted to obtain the relevant properties of $\Psi(z)$.

These include the basic property:

(P1) There exists $m_{0} \in \mathbb{Z}^{+}$such that for $m \geq m_{0}$ the series

$$
\sum_{V}\left|q_{V}(z)\right||(\alpha z+\beta)+i(\gamma z+\delta)|^{-2 m}
$$

converges uniformly on compact subsets of $\mathcal{H}$ and, in fact, on any strip of the form

$$
\{z|| \Re z \mid<1 / \varepsilon, \Im z>\varepsilon\}, \quad \varepsilon>0 .
$$

From (P1) we obtain the following directly.

(P2) If $f(z)$ is bounded in $\mathcal{H}$, and $m \geq m_{0}$, then

$$
\lim _{\Im z \rightarrow \infty} \Psi(z)=0 \text {. }
$$

(P3) If $m \geq m_{0}$ and $f(z)$ is bounded and holomorphic in $\mathcal{H}$, then $\Psi \in \mathcal{P}$.

It is important to point out that for the case when the underlying group is an H-group, the proof of the convergence of either (27) or (29) depends crucially upon Eichler's estimates of word length in an H-group [4, §3]. Since $G_{\lambda}, \lambda>2$, is Fuchsian of the second kind, Eichler's proof of these estimates does not apply to the case at hand. However, in Section 6, below, we provide an alternative proof of the required estimates tailored specifically to the groups $G_{\lambda}, \lambda>2$. With these estimates the proof of (P1) follows from the considerations of [10, pp. 615-619] and [11], together with calculations from [7, pp. 605-607]. In the appendix (Section 7) we give a proof of Eichler's 
estimates for arbitrary finitely generated groups of the second kind. The proof is patterned upon Eichler's in [4], but with significant changes.

As a consequence of absolute convergence, $\Psi(z)$ has the transformation property

$$
\left(\left.\Psi\right|_{k} ^{v} M\right)(z)=(\gamma z+\delta)^{2 m} \Psi(z)-(\gamma z+\delta)^{2 m} \vartheta_{m}(z) q_{M}(z)
$$

for $M=\left(\begin{array}{cc}* & * \\ \gamma & \delta\end{array}\right) \in G_{\lambda}$, where

$$
\vartheta_{m}(z)=\vartheta_{m}(f ; z)=\sum_{V} \frac{f(V z)}{[(\alpha z+\beta)+i(\gamma z+\delta)]^{2 m}} .
$$

In (32) the summation is over all $V=\left(\begin{array}{cc}\alpha & \beta \\ \gamma & \delta\end{array}\right) \in G_{\lambda}$.

For large $m \in \mathbb{Z}^{+}$, the series (32) converges absolutely-uniformly on compact subsets of $\mathcal{H}$ and, in fact, on sets of the form (30), provided $f(z)$ is bounded in $\mathcal{H}$. (Since $G_{\lambda}$ is finitely generated of the second kind, $m \geq 1$ is actually sufficient for absolute convergence.) Therefore, $\vartheta_{m}(z)$ is a holomorphic automorphic form on $G_{\lambda}$ of weight $2 m$ and multiplier system $\equiv 1$ :

$$
(\gamma z+\delta)^{-2 m} \vartheta_{m}(M z)=\vartheta_{m}(z),
$$

for all $M=\left(\begin{array}{cc}* & * \\ \alpha & \beta\end{array}\right) \in G_{\lambda}$. Defining

$$
F(z)=-\Psi\left(\left\{q_{M}\right\}, f, m ; z\right) / \vartheta_{m}(f ; z),
$$

we find that

$$
\left(\left.F\right|_{k} ^{v} M\right)(z)=F(z)+q_{M}(z), \quad M \in G_{\lambda} .
$$

In particular, $F(z+\lambda)=F(z)$ and $F(z)$ has the formal property (19) we seek in Theorem 2.

The difficulty remains that in order to complete the proof of Theorem 2 and hence of Theorem 1), we require that $F$ satisfy (17) and (18), in addition to (19). As we have already noted, (17) and (18) are jointly equivalent to the condition $F \in \mathcal{P}$. Now, by property (P3), above, we know that the numerator $\Psi$ is in $\mathcal{P}$ provided $m \geq m_{0}$ and $f$ is bounded and holomorphic in $\mathcal{H}$. On the other hand, the denominator $\vartheta_{m}$ may well have zeroes in $\mathcal{H}$; in this case $F$ could have poles in $\mathcal{H}$ and fail to be in $\mathcal{P}$. Clearly, it would be ideal to choose $f$, bounded and holomorphic in $\mathcal{H}$, so that $\vartheta_{m}(f ; z)$ is bounded away from zero in $\mathcal{H}$. While this is not possible, the results of [7] show that we can come close enough to the ideal to complete the proof of Theorem 2 .

5. Proof of Theorem 2. The key to the proof is the "Modified Main Lemma" of [7, p. 614], applicable here since $G_{\lambda}, \lambda>2$, is a finitely generated Fuchsian group of the second kind. This result, applied to the region $\mathcal{H}$, with conformal mapping $\varphi(z)=(-z+i) /(z+i)$ of $\mathcal{H}$ onto $\mathcal{U}$ (the unit disk), and the group $G_{\lambda}$ implies 
Lemma 3. Suppose $m \in \mathbb{Z}$, with $m \geq 2$. Then there exists a polynomial $p=p_{m}$ such that

(i) the Poincaré series

$$
\vartheta_{m}\left(p_{m} \circ \varphi ; z\right)=\sum_{V \in G_{\lambda}} \frac{p_{m}\left(\frac{-V z+i}{V z+i}\right)}{[(\alpha z+\beta)+i(\gamma z+\delta)]^{2 m}}
$$

has an expansion at $i \infty$ of the form

$$
\vartheta_{m}\left(p_{m} \circ \varphi ; z\right)=\sum_{n=1}^{\infty} c_{n} e^{2 \pi n z / \lambda}, \quad c_{1} \neq 0,
$$

valid for $z \in \mathcal{H}$

(ii) $|z+i|^{2 m}\left|\vartheta_{m}\left(p_{m} \circ \varphi ; z\right)\right|$ is bounded away from zero in the "truncated fundamental region" $\mathcal{R}_{\lambda}\left(y_{0}\right)=\left\{z \in \mathcal{R}_{\lambda} \mid \Im z<y_{0}\right\}$, for each $y_{0}>0$.

It follows immediately from (ii) that

(P4) $\left|\vartheta_{m}\left(p_{m} \circ \varphi ; z\right)\right|$ is bounded away from zero in $\mathcal{R}_{\lambda}\left(y_{0}\right)$, for each $y_{0}>0$.

We may now complete the proof of Theorem 2 by considering the function $\Psi(z)=\Psi\left(\left\{q_{M}\right\}, p_{m} \circ \varphi, m ; z\right)$, with $m \geq m_{0}$, where $m_{0}$ is defined in (P1) of Section 4. Then, as before, (31) holds and, with $F$ defined by

$$
F(z)=\frac{-\Psi\left(\left\{q_{M}\right\}, p_{m} \circ \varphi, m ; z\right)}{\vartheta_{m}\left(p_{m} \circ \varphi ; z\right)},
$$

as in (34), (35) holds as well. We claim that with the special choice $f=$ $p_{m} \circ \varphi$, Lemma 3 implies that $F \in \mathcal{P}$. If so, this establishes Theorem 2 , and hence Theorem 1.

It follows from (35), with $M=S$, that $\Psi(z+\lambda)=\Psi(z)$, because $q_{S}=0$. Thus since $\Psi$ is holomorphic in $\mathcal{H}$, (P2) of Section 4 implies that

$$
\Psi(z)=\sum_{n=1}^{\infty} d_{n} e^{2 \pi n z / \lambda}, \quad z \in \mathcal{H}
$$

Now (36), (38) and (P4) together imply that $F$ defined by (37) satisfies (28) for $z$ restricted to $\mathcal{R}_{\lambda}$. On the other hand, since $q_{M} \in \mathcal{P}$ for all $M \in G_{\lambda}$, the reasoning of $[9$, pp. 149-150] shows $F \in \mathcal{P}$.

6. Word length in $G_{\lambda}, \lambda>2$. Let $G_{\lambda}$ be a Hecke triangle group with $\lambda>2$. Let $A=\left(\begin{array}{ll}a & b \\ c & d\end{array}\right)$ and let $\mu(A)=a^{2}+b^{2}+c^{2}+d^{2}$. Then $A$ can be written as a word in the generators $S=S_{\lambda}$ and $T$ :

$$
A=S^{a_{1}} T S^{a_{2}} T \ldots T S^{a_{n}}, \quad a_{i} \in \mathbb{Z}, n \geq 2, a_{2}, \ldots, a_{n-1} \neq 0 .
$$

Define wl $A=n-1+\sum_{i=1}^{n}\left|a_{i}\right|$ and fl $A=n-1+l$ where $l$ is the number of $a_{i} \neq 0$. (Thus $l=n-2, n-1$ or $n$.) We call a word given by (39) normalized if $a_{1}, a_{n} \neq 0$. (In this case $\mathrm{fl} A=2 n-1$.) 
LEMMA 4. With the above notation,

(i) wl $A \leq c_{\lambda} \mu(A)$;

(ii) fl $A \leq d_{\lambda} \log \mu(A)$.

P r o of. Let $A$ be given by (39). We claim that without loss of generality $A$ may be assumed to be both hyperbolic and normalized. To guarantee that the word is normalized, we pass from $A$ to $A^{\prime}=A, T A, A T$, or $T A T$ and observe that $\mu(A)=\mu\left(A^{\prime}\right)$, in every case.

Next observe that $A \in G_{\lambda}$ is elliptic if and only if $A=\left(\begin{array}{cc}a & b \\ c-a\end{array}\right)$, that is, $\operatorname{tr} A=0$. Thus $A$ is hyperbolic if and only if $\operatorname{tr} A \neq 0, \pm 2$. Note further that $A$ has the form $\left(\begin{array}{ll}a & b \\ 0 & d\end{array}\right)$ if and only if $a=d= \pm 1$, that is, $A$ is a translation (and thus parabolic). From these facts it follows that at least one of the words $A^{\prime \prime}=S^{j} A^{\prime}$, with $j \in \mathbb{Z}$ and $|j| \leq 2$, is both normalized and hyperbolic.

Furthermore, calculation shows that $\mu\left(A^{\prime \prime}\right)<\left(1+\lambda|j|+\lambda^{2} j^{2}\right) \mu(A)$, wl $A^{\prime \prime} \geq \mathrm{wl} A^{\prime}-|j|=\mathrm{wl} A-s-|j|$ and $\mathrm{fl} A^{\prime \prime}=\mathrm{fl} A^{\prime}-t=\mathrm{fl} A-s-t$; here $t=0$ or 1 and $s=0,1$ or 2 . We conclude that wl $A^{\prime \prime} \geq \mathrm{wl} A-4$ and fl $A^{\prime \prime} \geq \mathrm{fl} A-3$, so that the proof has been reduced to the case of normalized and hyperbolic $A$.

For any $a>b$ and $c>0$ we have, by [1, p. 130, formula 7.2.1(i)],

$$
d(b+i c, a+i c)=\log \frac{(|b-a+2 i c|+|b-a|)^{2}}{4 c^{2}} \geq \log \left(\frac{(b-a)^{2}}{c^{2}}\right) .
$$

Let $0<c_{0}<1$ be the minimum height of the Nielsen convex region $(\mathcal{N})$ of $G_{\lambda}$. Let $\ell(A)=$ length of the geodesic that the axis of $A$ projects to on $G_{\lambda} \backslash \mathcal{H}$. That the geodesic must lie entirely in $\mathcal{N}$ is well known. This geodesic may be lifted to a fundamental set of $\operatorname{arcs}$ in $\mathcal{H}$ consisting of h-arcs emanating from the unit circle (above $c_{0}$ ) and returning to a translate (by $a_{j} \lambda$-right or left according as $a_{j}$ is positive or negative) of this circle, again above $c_{0}$.

If $\left|a_{j}\right| \geq 2$, the h-length of the entire arc associated with $a_{j}$ is $\geq \log \left(\lambda\left(\left|a_{j}\right|-1\right)\right)^{2}$, by inequality (40) with $b= \pm a_{j} \lambda \mp \frac{1}{2} \lambda, a= \pm \lambda / 2$, $c=1$. (That is, the quantity given by these choices is shorter than the piece of the axis of $A$ associated with $a_{j}$.) Note that $\left(\lambda\left(\left|a_{j}\right|-1\right)\right)^{2} \geq\left(\lambda a_{j} / 2\right)^{2}$.

If $\left|a_{j}\right|=1$, we must choose (by symmetry) an arc connecting the unit circle above $c_{0}$ with a point of the same height on the translate of the unit circle by $\pm \lambda$. Such an arc, again by the equality of (40), has length greater than or equal to $\log \left(1+(\lambda-2)^{2} / 2\right)$.

Thus we see that in both cases the $a_{j}$ portion of $\ell(A)$ has length at least $\log \left(1+e_{\lambda}\right)\left|a_{j}\right|^{2}$, where $e_{\lambda}$ is independent of $\left|a_{j}\right|$, and

$$
\ell(A) \geq n \log \left(1+e_{\lambda}\right)+\sum_{j=1}^{n} \log \left|a_{j}\right|^{2} .
$$


On the other hand, by [1, p. 173, bottom equation],

$$
|\operatorname{tr} A|=2 \cosh \left(\frac{1}{2} \ell(A)\right) \geq e^{\ell(A) / 2} .
$$

And so,

We now have:

$$
\ell(A) \leq 2 \log |\operatorname{tr} A|
$$

$$
n \log \left(1+e_{\lambda}\right)+\sum_{j=1}^{n} \log \left|a_{j}\right|^{2} \leq \ell(A) \leq 2 \log |\operatorname{tr} A| .
$$

Exponentiating, then taking the square root, we have

$$
\left(1+e_{\lambda}\right)^{n / 2} \prod_{j=1}^{n}\left|a_{j}\right| \leq|\operatorname{tr} A| .
$$

We are now at a juncture.

(1) The product on the LHS of (41) is at least one. Thus

$$
\left(1+e_{\lambda}\right)^{n / 2}<|\operatorname{tr} A|<\mu(A),
$$

the last inequality being obvious. Upon taking logarithms, we see that this implies (ii).

(2) We note that $\prod^{2}\left|a_{j}\right| \geq \sum^{2}\left|a_{j}\right|$ (where the ${ }^{2}$ indicates that only those terms with $\left|a_{j}\right| \geq 2$ are included). This gives

$$
\begin{aligned}
\text { wl } A & =n+\sum\left|a_{j}\right| \leq 2 n+\sum^{2}\left|a_{j}\right| \leq 2 n+\left(1+e_{\lambda}\right)^{n / 2} \prod^{2}\left|a_{j}\right| \\
& <2 n+|\operatorname{tr} A|<c_{\lambda} \mu(A) .
\end{aligned}
$$

(The last inequality follows from the logarithmic bound on $n$ in terms of $\mu(A)$ established by proving (ii).)

This ends the proof of Lemma 4.

LEMMA 5. By a terminal sequence of a word $A=B_{1} B_{2} \ldots B_{n}$ in the generators $B_{i}$ of a group we mean the product $B_{k} \ldots B_{n}$ for some $1 \leq k \leq n$. Then, with $A \in G_{\lambda}$ expressed in terms of the generators $S$ and $T$, we have $\mu($ terminal sequence of $A) \leq c \mu(A)$.

Proof (by induction). Let $T_{n}$ be the $n$th terminal sequence. The lemma is obvious if $n=1$, as then $T_{1}=S$ or $T$. Next,

$$
T_{n+1}= \begin{cases}T \cdot T_{n}, & \text { or } \\ S^{ \pm 1} \cdot T_{n} .\end{cases}
$$

Now $\mu\left(T \cdot T_{n}\right)=\mu\left(T_{n}\right)$, so that case is no problem. We need to prove the following

Simple FACT. Keep track of $T_{n}(i)$. At the moment after we apply a $T$, $T_{n}(i)$ is in the unit circle $\mathcal{U}$. 
Proof of Simple Fact (by induction). First, $T S^{ \pm m}(i) \in \mathcal{U}$. Next, $T S^{ \pm m} T(i)=T S^{ \pm m}(i) \in \mathcal{U}$. Since $S^{ \pm m}(\mathcal{U}) \subset \mathcal{H} \backslash \mathcal{U}$, we see that the next application of $T$ takes us back to $\mathcal{U}$.

It follows from the Simple Fact that the orbit of $i$ (as we go through the terminal sequences in order) bounces into $\mathcal{U}$ by virtue of an application of $T$, and then leaves $\mathcal{U}$ moving monotonically further to the right or left depending on whether we are working through an application of a positive or negative power of $S$.

This says that $\left|S^{ \pm 1} T_{n}(i)\right|>\left|T_{n}(i)\right|$, if the LHS is the next terminal sequence term. In terms of matrices, the lower rows of $B$ and $S^{ \pm 1} \cdot B$ are the same. Let $T_{n}=\left(\begin{array}{l}a b \\ c d\end{array}\right)$. Obviously $\left|T_{n}(i)\right|^{2}=\left(|a|^{2}+|b|^{2}\right) /\left(|c|^{2}+|d|^{2}\right)$, so the inequality at the head of this paragraph and the identity of the two lower rows forces $\left(|a \pm \lambda c|^{2}+|b \pm \lambda c|^{2}\right)>\left(|a|^{2}+|b|^{2}\right)$, where the sign is chosen the same as the sign of the power of $S$.

We have shown that $\mu\left(T_{n}\right)$ is increasing with $n$, and strictly increasing except when the applications of $T$ are executed.

This ends the proof of (rather more than) Lemma 5.

\section{Appendix: Word length for arbitrary finitely generated Fuchsian groups of infinite volume}

7.1. Eichler's "Hilfssätze 1 und 2" for groups of finite volume. At this point there is an abrupt shift in the written style of this paper from the formal one customary in number theory (especially analytic) to the looser one of geometry (especially differential). This reflects the style chosen by Eichler for the geometric portions of [4], which this section exposes and extends.

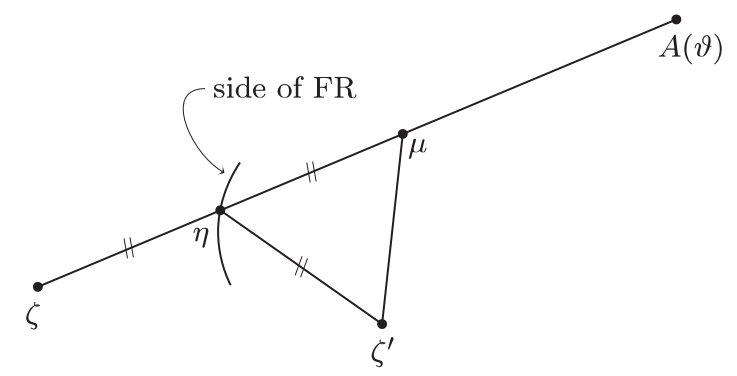

Picture 1

Given an element $A$ of a finitely generated Fuchsian group of the first kind, Eichler's goal is to estimate wl $A$ for a particular word for $A$ in the group generators (called $B$ 's below) gotten from the side pairing of the Dirichlet fundamental region $\mathcal{R}$ for the group. In essence his idea is this: 
draw a geodesic between $\mathcal{R}$ and $A(\mathcal{R})$, and keep track of which sides are crossed in moving towards $A(\mathcal{R})$ from $\mathcal{R}$, to get a word for $A$. Also, the length of the geodesic (measured in units of the diameter of $\mathcal{R}$ ) should be about the word-length of this word for $A$. There are two problems here: (i) one can pass through many fundamental regions (thus adding appreciable word-length) without adding much length, if the geodesic runs near (actually, around and around) a cusp; (ii) likewise, if the geodesic runs near an elliptic fixed point of high order. (It is easy to choose a geodesic that does not go through an elliptic fixed point, which is geometrically rather intractable unless that fixed point has order 2.)

Eichler cleverly handles these problems by dynamically (or recursively) altering the geodesic as follows. Let $\zeta$ be the center of (the Dirichlet fundamental region) $\mathcal{R}$. He chooses an initial geodesic connecting $\zeta$ to the point in $A(\mathcal{R})$ equivalent to some $\vartheta \in \mathcal{R}$, that is, $A(\vartheta)$. As the geodesic passes from $\mathcal{R}$ to the next fundamental region (whose center is called $\zeta^{\prime}$ ), we switch geodesics to the one connecting $\zeta^{\prime}$ to $A(\vartheta)$. It is a consequence of Eichler's proof that this process terminates, in a finite number of steps, at $A(\vartheta)$. This forces the sequence of crossings to produce a word for $A$.

Since elliptic fixed points are "far from" the center of $\mathcal{R}$, this adjustment solves the "additional word-length but no appreciable additional geodesic length" problem in this case. It does not do so for cusps, however, because $\vartheta$ may be "near" a cusp (high in the fundamental horocycle), and a uniform (in $\vartheta$ ) bound on how much geodesic length is added by each additional application of the parabolic is, of course, impossible. (This is equivalent to saying geodesic loops about cusps may have arbitrarily small length.) Eichler solves this problem by defining it away-not counting long runs of a single parabolic in his word-length, giving rise to the flat-length function introduced in Section 6 .

We are going to examine the word produced by the Eichler process in the case of finitely generated Fuchsian groups of the first kind. In particular, we will prove two things; first, that a crossing produced by a generator $B$ in the Eichler word for $A$ that is not parabolic and not the last letter of the word - such a crossing replaces $\zeta$ with $\zeta^{\prime}$ - has the property that $|\zeta-A(\vartheta)|-c>\left|\zeta^{\prime}-A(\vartheta)\right|$ for some $c$ independent of $\vartheta$ and $A$. Second, we will show that in the above picture if the crossing is caused by a $B_{k}$ that is parabolic, then there is an $\varepsilon$ independent of $\vartheta$ and $A$ such that $|\zeta-A(\vartheta)|<\left|\zeta^{\prime}-A(\vartheta)\right|+\varepsilon$ implies we must have $B_{k+1}=B_{k}$.

Consider the triangle $\mu, \eta, \zeta^{\prime}$ (Picture 1). If side $\mu \zeta^{\prime}$ is less than twice either of the other sides by a definite amount (here and below this means by a constant amount depending only on the group) with respect to $\vartheta$ and $A$, then we have our first result. If this is false it must be possible to choose $A$ and $\vartheta$ so that this is an isosceles triangle with base angles arbitrarily 
close to zero. Thus $\left|\zeta-\zeta^{\prime}\right|$ will be arbitrarily small, because $\eta$ moves along a finite range, the point being that the triangle has bounded size, so the small base angles force $\zeta$ and $\zeta^{\prime}$ together. (This would not be so if $\eta$ were permitted to go to $\infty$ (hyperbolically), an eventuality we will analyze when $B$ is parabolic.) However, $\left|\zeta-\zeta^{\prime}\right|$ is bounded below, as they are centers of fundamental region and the group is finitely generated. Note that this argument fails if $A(\vartheta)$ is nearer to $\eta$ than $\zeta$ is - so that we cannot construct $\mu$. This is surely possible if we are at the last letter (crossing) of the word and might even happen at the last "few" crossings. However, the word-length estimates that Eichler proves based on these results are unaffected as we really only need to know how many additional letters accrue in the word once this phenomenon occurs. The number is less than the eccentricity of the fundamental region - the ratio of longest to shortest amongst distances from center to sides of the fundamental region.

Now if $B$ is parabolic and the angles go to zero as $\eta$ goes to cusp $p$, our first observation is that $A(\vartheta)$ is going to the same cusp. This is easily seen by moving $p$ to $\infty$ (Picture 2).

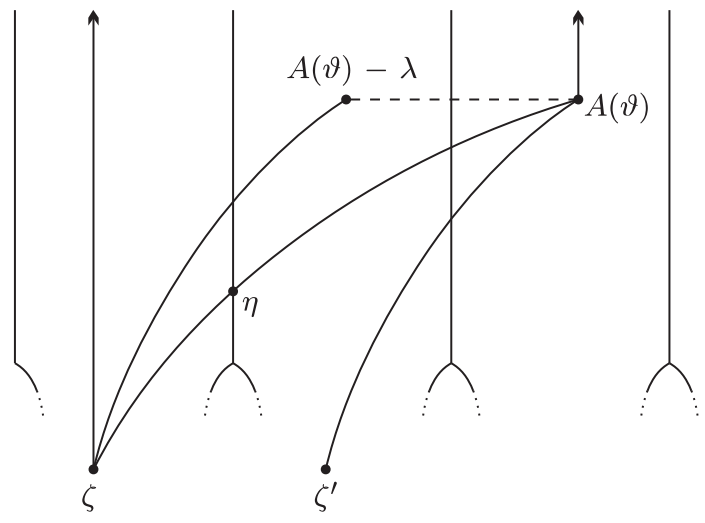

Picture 2

In the illustration, the h-line between $\zeta$ and $p$ (drawn as $\infty$ here) hits no other fundamental region side (by convexity). By hypothesis $\eta$ is chosen large enough so that the h-line between $\zeta$ and $\eta$ hits the boundary of the fundamental region first at $\eta$, which lies on a side fixed by $p$. (There is an obvious abuse of language here.)

Connectedness of the boundary of the fundamental region now ensures that the entire quadrilateral $\infty \zeta A(\vartheta) \infty$ contains no piece of a side of the fundamental region not fixed by $p$. The line between $\zeta^{\prime}$ and $A(\vartheta)$ is equivalent to the line between $\zeta$ and $A(\vartheta)-\lambda$, where $\lambda$ is the translation length for $p$. But this line lies in the quadrilateral. We have shown that for $\eta$ (and any point with imaginary part above $\Im \eta), B_{k}=B_{k+1}=$ the parabolic fixing $p$. 
We get the formulation of the second result above if we choose $\varepsilon$ to be the length of a small loop (well) within the fundamental horocycle at $\infty$ (see the dotted line in Picture 2).

7.2. Observation. Note that the estimates of Section 6 are valid for all words in $S$ and $T$ whereas the Eichler estimate only applies to the Eichler word for $A$. It is unclear whether other words for $A$ just as worthy (i.e., not trivially inflated in length by subwords that are the identity or by not being cyclically reduced) enjoy Eichler estimates. Still Eichler's is an early and fine effort in what has come to be known as the theory of automatic groups.

7.3. Eichler's argument for finitely generated groups of the second kind. In our context it is natural to ask how much of Eichler's approach (that is, his method of generating a word for $A$ ) can be carried over to finitely generated groups of the second kind. The answer is simple: everything can be made to work perfectly. There are (at least) two available routes to take.

7.3.1. The Nielsen convex region approach. Here we would begin the Eichler algorithm with the following two intrinsic (in the sense of differential geometry) restrictions: we require both $\zeta$, the center of our fundamental region, and $\vartheta$, our target point, to lie in $\mathcal{N}$ (the Nielsen convex region). In this case it is easy to see, using the well-known fact that any geodesic leaving $\mathcal{N}$ never returns to it, that all the arcs in Eichler Pictures 1 and 2 lie entirely in $\mathcal{N}$. (We could just place the restriction on $\vartheta$ and consider geodesics all of which begin outside the Nielsen region, but then enter it (once only), terminating at $\vartheta$.)

This approach means that when $\eta$ lies on a side of the fundamental region paired by a hyperbolic fixing the boundary geodesic (there must be such a hyperbolic for each free side), it must lie on the portion of this side within $\mathcal{N}$. This insures that the compactness argument of Eichler succeeds.

7.3.2. The direct approach. We can also study the analog of Eichler Picture 2 in the case of a free side (Eichler Picture 3a, b).

What we shall find is this: for any $\vartheta$ outside $\mathcal{N}$, the conclusion of Hilfssatz 1 applies. That is,

$$
|\zeta-A(\vartheta)|-c>\left|\zeta^{\prime}-A(\vartheta)\right| .
$$

First of all, note that we are assuming that $\zeta^{\prime}=B(\zeta)$, where $B$ is the hyperbolic fixing the boundary geodesic (whose length we will call $\partial$ ). Next, clearly we have $\left|\zeta^{\prime}-A(\vartheta)\right|<|\zeta-A(\vartheta)|<\left|\zeta^{\prime}-A(\vartheta)\right|+\partial$. The latter inequality holds because, as we did in the parabolic case, we can translate $\zeta^{\prime} A(\vartheta)$ back to $\zeta$ by $B$, proceed along this translate from $\zeta$ to the boundary geodesic, traverse the boundary geodesic once and then complete the trip to $A(\vartheta)$ via 


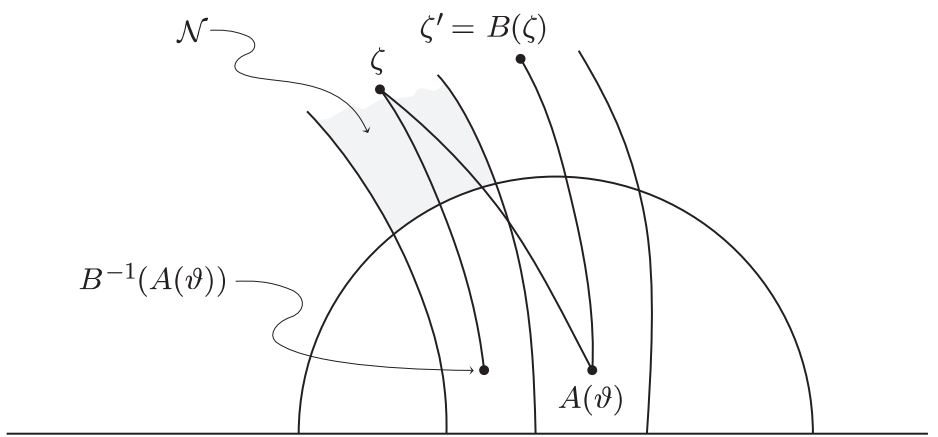

Picture 3a

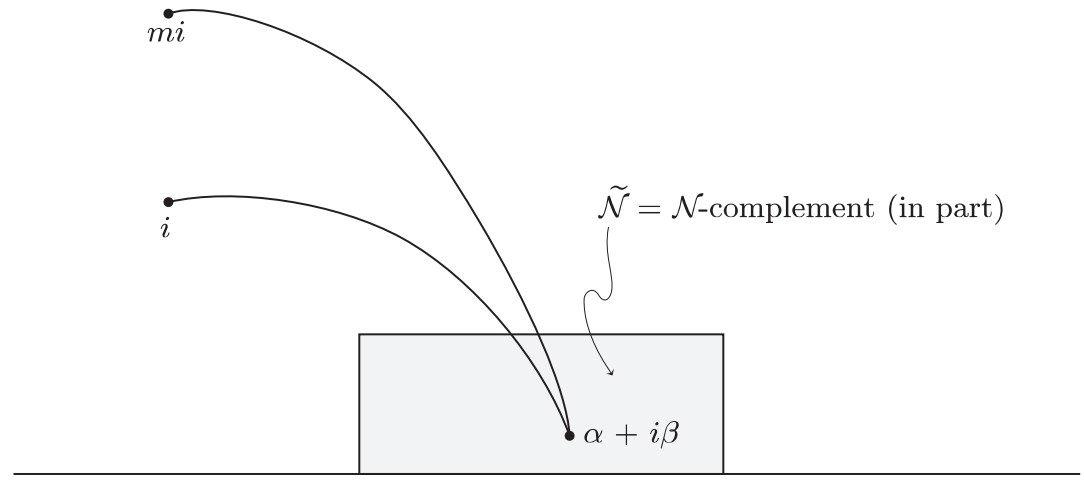

Picture $3 b$

the original geodesic from $\zeta^{\prime}$. This shows that the distances from $\zeta$ and $\zeta^{\prime}$ to $A(\vartheta)$ differ by a bounded amount.

(Aside: The analogous reasoning in the parabolic case shows why Eichler needed Hilfssatz 2. In effect, the boundary geodesic has length which is arbitrarily small and so the inequality in the previous paragraph shows that Hilfssatz 1 fails.)

In fact, these two distances differ by a definite amount, if we restrict consideration to $\vartheta$ outside $\mathcal{N}$. To see this, calculate the hyperbolic distance between $i$ and $\alpha+\beta i$, the latter point being constrained to lie in portion of a finite rectangle lying on the real axis of height less than one by a definite amount. Next, repeat the calculation replacing the first point $i$ with $m i, m>1$. Last, note that the difference of these two distances is uniformly bounded and bounded away from zero even though $\beta$ is permitted to approach zero. Because we may assume $\zeta$ and $\zeta^{\prime}$ are a definite distance from $\mathcal{N}$, and they can be mapped to $i$ and $m i$ by an isometry preserving that distance, we are done. 


\section{References}

[1] A. Beardon, The Geometry of Discrete Groups, Grad. Texts in Math. 91, Springer, New York, 1983.

[2] S. Bochner, Some properties of modular relations, Ann. of Math. 53 (1951), 332-363.

[3] K. Chandrasekharan and R. Narasimhan, Hecke's functional equation and arithmetical identities, ibid. 74 (1961), 1-23.

[4] M. Eichler, Grenzkreisgruppen und kettenbruchartige Algorithmen, Acta Arith. 11 (1965), 169-180.

[5] H. Hamburger, Über die Riemannsche Funktionalgleichung der $\zeta$-Funktion, Math. Z. 10 (1921), 240-254.

[6] E. Hecke, Herleitung des Euler-Produktes der Zetafunktion und einiger L-Reihen aus ihr Funktionalgleichung, Math. Ann. 119 (1944), 266-287.

[7] M. Knopp, A corona theorem for automorphic forms and related results, Amer. J. Math. 91 (1969), 599-618.

[8] —, On Dirichlet series satisfying Riemann's functional equation, Invent. Math. 117 (1994), 361-372.

[9] - On the growth of entire automorphic integrals, Results in Math. 8 (1985), 146-152.

[10] - Some new results on the Eichler cohomology of automorphic forms, Bull. Amer. Math. Soc. 80 (1974), 607-632.

[11] J. Lehner, Automorphic forms with preassigned periods, J. Res. Nat. Bur. Standards Sect. B 73 (1969), 153-161.

[12] C. L. Siegel, Bemerkung zu einem Satz von Hamburger über die Funktionalgleichung der Riemannschen Zetafunktion, Math. Ann. 86 (1922), 276-279.

Temple University

Philadelphia, Pennsylvania 19022

U.S.A.
CUNY, Baruch College New York, New York 10010

U.S.A.

E-mail: marksh@panix.com 